\title{
DNA methylation and cognitive functioning in healthy older adults
}

\author{
Olga J. G. Schiepers ${ }^{1 *}$, Martin P. J. van Boxtel $^{1}$, Renate H. M. de Groot ${ }^{1,2,3}$, Jelle Jolles ${ }^{1,2}$, Frans J. Kok $^{4}$, \\ Petra Verhoef ${ }^{4,5,6}$ and Jane Durga ${ }^{4,5,7}$ \\ ${ }^{1}$ Department of Psychiatry and Neuropsychology, School for Mental Health and Neuroscience (MHeNS)/European Graduate \\ School of Neuroscience (EURON), Maastricht University/Maastricht University Medical Centre, PO Box 616, 6200 MD \\ Maastricht, The Netherlands \\ ${ }^{2}$ Faculty of Psychology and Education, AZIRE Research Institute, VU University Amsterdam, Amsterdam, The Netherlands \\ ${ }^{3}$ Centre for Learning Sciences and Technologies, Open University, Heerlen, The Netherlands \\ ${ }^{4}$ Division of Human Nutrition, Wageningen University, Wageningen, The Netherlands \\ ${ }^{5}$ Top Institute Food and Nutrition, Wageningen, The Netherlands \\ ${ }^{6}$ Unilever Research and Development, Vlaardingen, The Netherlands \\ ${ }^{7}$ Cognitive Sciences Group, Nutrition and Health Department, Nestlé Research Centre, Lausanne, Switzerland \\ (Received 4 March 2011 - Revised 23 May 2011 - Accepted 2 June 2011 - First published online 27 July 2011)
}

\begin{abstract}
Long-term supplementation with folic acid may improve cognitive performance in older individuals. The relationship between folate status and cognitive performance might be mediated by changes in methylation capacity, as methylation reactions are important for normal functioning of the brain. Although aberrant DNA methylation has been implicated in neurodevelopmental disorders, the relationship between DNA methylation status and non-pathological cognitive functioning in human subjects has not yet been investigated. The present study investigated the associations between global DNA methylation and key domains of cognitive functioning in healthy older adults. Global DNA methylation, defined as the percentage of methylated cytosine to total cytosine, was measured in leucocytes by liquid chromatography-MS/MS, in 215 men and women, aged 50-70 years, who participated in the Folic Acid and Carotid Intima-Media Thickness (FACIT) study (clinical trial registration number NCT00110604). Cognitive performance was assessed by means of the Visual Verbal Word Learning Task, the Stroop Colour-Word Interference Test, the Concept Shifting Test, the Letter-Digit Substitution Test and the Verbal Fluency Test. Using hierarchical linear regression analyses adjusted for age, sex, level of education, alcohol consumption, smoking status, physical activity, erythrocyte folate concentration and 5,10-methylenetetrahydrofolate reductase $677 \mathrm{C} \rightarrow \mathrm{T}$ genotype, we found that global DNA methylation was not related to cognitive performance on any of the domains measured. The present study results do not support the hypothesis that global DNA methylation, as measured in leucocytes, might be associated with cognitive functioning in healthy older individuals.
\end{abstract}

Key words: DNA methylation: Epigenetics: Cognitive performance: Population-based studies

Most cognitive functioning declines with advancing age, and identifying the risk factors for age-related cognitive decline has become a topic of increasing interest. Previous research has indicated that a low folate status might increase the risk of cognitive impairment ${ }^{(1)}$. However, the potential biological mechanisms underlying this relationship remain to be elucidated.

One possible mechanism that might explain the involvement of folate status in cognitive performance is DNA methylation, which refers to the epigenetic modification of gene expression by the addition of methyl groups to cytosine residues in $\mathrm{DNA}^{(2)}$. Recent studies on animals have suggested that DNA methylation may be involved in regulating synaptic plasticity in hippocampal neurons, thereby influencing learning and memory processes ${ }^{(3,4)}$. In humans, both hypomethylation and hypermethylation of DNA have been implicated in psychiatric disorders, including schizophrenia ${ }^{(5)}$; neurodegenerative disorders, such as Alzheimer's disease ${ }^{(6)}$; and syndromes associated with mental retardation, e.g. Fragile X syndrome ${ }^{(7)}$.

Methyl groups for DNA methylation are provided by the universal methyl donor $S$-adenosylmethionine, which is synthesised from methionine ${ }^{(8)}$. Folic acid may increase the availability of $S$-adenosylmethionine by promoting the conversion of homocysteine into methionine, thereby influencing DNA methylation status ${ }^{(9)}$. Indeed, an intervention study in

Abbreviations: FACIT, Folic Acid and Carotid Intima-Media Thickness (FACIT); MTHFR, 5,10-methylenetetrahydrofolate reductase.

*Corresponding author: O. J. G. Schiepers, fax +31 433884092, email olga.schiepers@maastrichtuniversity.nl 
older women has shown that low dietary folate intake was associated with global DNA hypomethylation, which could be reversed by folate repletion ${ }^{(10)}$. In addition, the common 5,10-methylenetetrahydrofolate reductase (MTHFR) $677 \mathrm{C} \rightarrow \mathrm{T}$ polymorphism, which mimics folate deficiency by impairing the conversion of homocysteine into methionine, has also been related to DNA hypomethylation ${ }^{(11)}$.

Given the role of folate metabolism in generating methyl donors for methylation processes, and the involvement of DNA methylation in brain functioning, it seems reasonable to hypothesise that folate status might influence cognitive functioning by exerting effects on DNA methylation. However, the association between DNA methylation status and cognitive performance in the general population has not yet been investigated. Therefore, the present study examined whether leucocyte global DNA methylation was associated with cognitive performance in healthy older adults.

\section{Methods}

\section{Study population}

The present study was performed using data from the Folic Acid and Carotid Intima-Media Thickness (FACIT) study, a randomised, double-blinded, placebo-controlled trial, originally designed to investigate the effects of 3-year folic acid supplementation on the risk of $\mathrm{CVD}^{(12)}$. The study population consisted of 818 healthy men and women, aged $50-70$ years at baseline. A detailed description of the study design and the selection of participants can be found elsewhere ${ }^{(12)}$.

Venous blood samples were collected at baseline. Leucocyte global DNA methylation was determined in a sub-sample of 216 participants. First, the study population was stratified by MTHFR $677 \mathrm{C} \rightarrow \mathrm{T}$ genotype, to ensure equal distribution of MTHFR $677 \mathrm{C} \rightarrow \mathrm{T}$ genotypes in the final sample. Thereafter, participants in the folate treatment group were randomly selected from the three strata and were individually matched with participants in the placebo group on the variables age, sex, smoking status and MTHFR $677 \mathrm{C} \rightarrow \mathrm{T}$ genotype, as these variables may influence DNA methylation ${ }^{(11,13,14)}$. Some samples were not measured due to human error in sample retrieval. Valid DNA methylation data were available for 111 participants in the treatment group and 105 participants in the placebo group. As valid data on cognitive functioning were lacking for one participant in the folate treatment group, the final study sample consisted of 215 individuals.

This study was conducted according to the guidelines laid down in the Declaration of Helsinki and all procedures involving human participants were approved by the Medical Ethics Committee of Wageningen University. Written informed consent was obtained from all the participants.

\section{Cognitive functioning}

Cognitive functioning on the domains of memory, sensorimotor speed, complex speed, information processing speed and word fluency was assessed by a comprehensive neuropsychological test battery, consisting of the Visual Verbal
Word Learning Task, the Stroop Colour-Word Interference Test, the Concept Shifting Test, the Letter-Digit Substitution Test and the Verbal Fluency Test, as described earlier ${ }^{(12)}$.

\section{DNA methylation status and genotyping}

Genomic DNA was isolated from peripheral blood leucocytes at baseline. Global DNA methylation was determined by liquid chromatography-MS/MS, as described previously ${ }^{(15)}$. Genomic DNA methylation status was calculated as the percentage of methylated cytosine to total cytosine (mCyt/tCyt) using the following formula ${ }^{(15)}$ : (nmol $\mathrm{mCyt} /(\mathrm{nmol} \mathrm{mCyt}+\mathrm{nmol}$ Cyt)) $\times 100 \%$.

MTHFR $677 \mathrm{C} \rightarrow \mathrm{T}$ genotype was determined by PCR with restriction fragment length polymorphism analysis with $\operatorname{Hin} \mathrm{fI}^{(16)}$, and was defined as a common variant (CC or CT genotype) or a rare variant (TT genotype).

\section{Blood measurements}

Fasting venous blood samples were collected at baseline, processed directly and stored at $-80^{\circ} \mathrm{C}$. Serum folate was measured using a chemiluminescent immunoassay (Diagnostic Products Corporation, Los Angeles, CA, USA). Erythrocyte folate was determined in duplicate and the average was taken to reduce measurement error. Erythrocyte folate concentrations were calculated using the following formula: (unadjusted erythrocyte folate/haematocrit $)-((1-$ haematocrit $) /$ haematocrit $) \times$ serum folate. Plasma total homocysteine was determined by HPLC and fluorimetric detection, as described previously ${ }^{(17)}$.

\section{Demographic and lifestyle variables}

Level of education (low/middle/high) was measured by classifying formal schooling according to the Dutch educational system $^{(18)}$. Alcohol consumption (g/d) and current smoking (yes/no) were ascertained by means of self-report questionnaires. BMI $\left(\mathrm{kg} / \mathrm{m}^{2}\right)$ was calculated from height and weight, and physical activity was estimated using the Physical Activity Scale for the Elderly ${ }^{(19)}$.

\section{Statistical analysis}

Normality of data distributions was ascertained by normal $P-P$ plots. Baseline data were used to assess the cross-sectional associations between global DNA methylation status and cognitive functioning. Independent samples $t$ tests and univariate ANOVA were carried out to examine whether DNA methylation status varied according to sex, level of education, smoking status or MTHFR $677 \mathrm{C} \rightarrow \mathrm{T}$ genotype.

Hierarchical linear regression analyses were performed for DNA methylation status in relation to each of the five cognitive performance indices. The analyses were corrected for sociodemographic and lifestyle variables that were considered potential confounders, i.e. age, sex, level of education, alcohol consumption, smoking status, physical activity, erythrocyte folate concentration and MTHFR $677 \mathrm{C} \rightarrow \mathrm{T}$ genotype $^{(11,13,14,20)}$. 
To investigate the possibility of a non-linear relationship between global DNA methylation and cognitive performance, we repeated the analyses with the quadratic term for DNA methylation status as the independent variable, adjusted for covariates and the linear term for DNA methylation status. The quadratic term for DNA methylation status was expressed as the residuals of regressing (DNA methylation) ${ }^{2}$ on DNA methylation, i.e. the quadratic component that is orthogonal to the linear component of DNA methylation.

The statistical power for detecting associations between DNA methylation status and each of the dependent variables, assuming a small effect size of $f^{2}=0 \cdot 03$, was $0 \cdot 80$. Statistical differences were considered significant at $P<0 \cdot 05$. All analyses were performed using SPSS 16.0 (SPSS, Inc., Chicago, IL, USA).

\section{Results}

Table 1 summarises the characteristics of the study population. The percentage of methylated cytosine to total cytosine residues in leucocyte DNA ranged from 4.0 to $5.6 \%$, which was comparable to the range reported by other population-based studies $^{(11,15)}$. The extent of global DNA methylation did not vary according to sex $(t=-1 \cdot 285, P=0 \cdot 200)$, level of education $(F=0.611, P=0.544)$, smoking status $(t=1.611, P=0 \cdot 109)$ or MTHFR $677 \mathrm{C} \rightarrow$ T genotype $(t=-0.907, P=0 \cdot 365)$.

Hierarchical linear regression analyses corrected for age, sex, level of education, alcohol consumption, smoking status, physical activity, erythrocyte folate concentration and MTHFR $677 \mathrm{C} \rightarrow \mathrm{T}$ genotype did not reveal any significant associations between leucocyte global DNA methylation and cognitive performance on any of the domains measured (Table 2). In addition, repeating the analyses with the quadratic term for DNA methylation status as the independent variable did not yield any significant results (data not shown), implying that global DNA methylation did not show a non-linear relationship with cognitive performance.

\section{Discussion}

The present study did not support the hypothesis that individual variation in cognitive functioning in older adults might be related to the extent of leucocyte global DNA methylation.
Although there are no previous studies investigating the relationship between global DNA methylation and cognitive functioning in healthy human subjects, aberrant DNA methylation has been implicated in neurodevelopmental disorders ${ }^{(7)}$, psychiatric diseases $^{(5)}$ and neurodegenerative disorders ${ }^{(6)}$. In addition, animal research has suggested that DNA methylation status may be involved in learning and memory processes, e.g. by regulating synaptic plasticity in hippocampal neurons ${ }^{(3,4)}$.

The observed lack of a relationship between global DNA methylation and cognitive performance in healthy adults might imply that there is no functional relationship between the extent of cytosine methylation within DNA and individual differences in cognitive performance in the general population. In line with earlier reports ${ }^{(15)}$, we observed that global DNA methylation has a relatively narrow distribution in healthy individuals. These findings suggest that under non-pathological conditions, there appears to be little interindividual variation in DNA methylation-based regulation of gene expression, which decreases the likelihood that individual differences in cognitive performances may be mediated by this epigenetic mechanism.

Although global DNA methylation might not be involved in cognitive functioning, the present results do not rule out the possibility that DNA methylation at specific loci may be related to cognitive performance. In human subjects, gene-specific alterations in DNA methylation patterns have been associated with a number of pathological conditions characterised by cognitive deficits. Animal studies have suggested that dietinduced folate deficiency may result in overexpression of the Presenilin 1 gene by causing hypomethylation of its promoter region $^{(21)}$. Increased expression of this gene, which leads to elevated production of $\beta$-amyloid peptide, has been implicated in the aetiology of Alzheimer's disease ${ }^{(22)}$. In addition, schizophrenia has been associated with reduced expression of the gene encoding the protein Reelin, which is involved in neurodevelopment and synaptic plasticity, due to hypermethylation of the gene's promoter region ${ }^{(5)}$. However, although it may be speculated that gene-specific changes in DNA methylation might underlie part of the individual differences in non-pathological cognitive functioning, little is known

Table 1. Characteristics of the study population

(Mean values and $95 \%$ confidence intervals)

\begin{tabular}{|c|c|c|}
\hline Characteristics & Total sample ( $n$ 215) & $95 \% \mathrm{Cl}$ \\
\hline Age (years) & $60 \cdot 9$ & $60 \cdot 2,61 \cdot 6$ \\
\hline Female sex (\%) & 34.9 & \\
\hline Level of education (\% low/middle/high) & $26 \cdot 0 / 39 \cdot 1 / 34 \cdot 9$ & \\
\hline Alcohol consumption $(\mathrm{g} / \mathrm{d})^{\star}$ & $12 \cdot 6$ & $4 \cdot 5,23 \cdot 5$ \\
\hline Current smoker (\%) & 14.9 & \\
\hline BMI $\left(\mathrm{kg} / \mathrm{m}^{2}\right)$ & $26 \cdot 7$ & $26 \cdot 2,27 \cdot 2$ \\
\hline Physical activity (PASE score) & $149 \cdot 2$ & $140 \cdot 5,158 \cdot 0$ \\
\hline Erythrocyte folate $(\mathrm{nmol} / \mathrm{l})$ & $716 \cdot 0$ & $681 \cdot 2,750 \cdot 8$ \\
\hline Plasma total homocysteine $(\mu \mathrm{mol} / \mathrm{l})$ & 13.4 & $12 \cdot 9,13 \cdot 8$ \\
\hline MTHFR 677 C $\rightarrow$ T genotype (\% CC/CT/TT) & $34 \cdot 9 / 32 \cdot 6 / 32 \cdot 6$ & \\
\hline Leucocyte global DNA methylation status $(\%) \dagger$ & 4.6 & $4 \cdot 6,4 \cdot 7$ \\
\hline
\end{tabular}

PASE, Physical Activity Scale for the Elderly; MTHFR, 5,10-methylenetetrahydrofolate reductase.

${ }^{*}$ Median (interquartile range) is given because of skewed data distribution.

†Percentage of methylated to total cytosine. 
Table 2. Cross-sectional associations between leucocyte global DNA methylation and cognitive performance in older adults (Regression coefficients and $95 \%$ confidence intervals)

\begin{tabular}{|c|c|c|c|c|c|c|}
\hline Cognitive performance indices ${ }^{\star}$ & $R^{2}$ (step 1)† & $P$ & $R^{2}$ change (step 2$) \dagger$ & $\beta \ddagger$ & $95 \% \mathrm{Cl}$ & $P$ \\
\hline Memory & 0.201 & $<0.001$ & 0.006 & 0.35 & $-0.19,0.90$ & 0.204 \\
\hline Sensorimotor speed & 0.231 & $<0.001$ & 0.006 & 0.30 & $-0.16,0.75$ & 0.202 \\
\hline Complex speed & 0.197 & $<0.001$ & 0.000 & -0.08 & $-0.60,0.43$ & 0.755 \\
\hline Information processing speed & 0.212 & $<0.001$ & 0.000 & 0.10 & $-0.48,0.68$ & 0.736 \\
\hline Word fluency & 0.141 & $<0.001$ & 0.008 & 0.43 & $-0.18,1.03$ & 0.164 \\
\hline
\end{tabular}

${ }^{*}$ Cognitive performance indices are expressed as $Z$-scores.

$\dagger R^{2}$ represents the proportion of explained variance and $R^{2}$ change represents the change in the proportion of explained variance after each step in hierarchical linear regression analyses. The covariates age, sex, level of education, alcohol consumption, smoking status, physical activity, erythrocyte folate concentration and 5,10-methylenetetrahydrofolate reductase $677 \mathrm{C} \rightarrow \mathrm{T}$ genotype were entered in step 1 and DNA methylation status in step 2.

‡Unstandardised regression coefficient and $95 \% \mathrm{Cl}$ for DNA methylation status in step 2 .

about the genetic correlates of cognitive performance in healthy human subjects.

An alternative explanation for the present null findings is that cognitive performance might be related to short-term changes, i.e. within the range of hours, in DNA methylation patterns rather than individual variation on the level of global DNA methylation. Indeed, animal studies have reported that dynamic and reversible changes in DNA methylation, such as the transient methylation and demethylation of DNA, are crucial for synaptic plasticity, learning and memory processes $^{(3,4)}$. It might be complicated, however, to measure such short-term changes in DNA methylation in volunteers, which makes it rather difficult to test this possibility.

From a methodological perspective, the present study was limited by its cross-sectional nature. In addition, the fact that we determined global DNA methylation in leucocytes rather than brain tissue should also be considered a limitation, as the extent of DNA methylation might differ between cells derived from the periphery and the brain ${ }^{(23)}$. However, no direct measures of DNA methylation status in the central nervous system were available, given the inability to measure cerebrospinal fluid or brain DNA methylation status in volunteers.

It might also be argued that because of the relatively small sample size, the present study might have been underpowered to detect very modest associations. However, it should be noted that the present study had $80 \%$ power to detect a $3 \%$ change in the proportion of explained variance, which may be considered a small effect size ${ }^{(24)}$.

The present study did not support the notion that folate metabolism might influence cognitive performance through the mechanism of global DNA methylation, as measured in leucocytes. In line with the present findings, we found that long-term supplementation with folic acid, which significantly improved cognitive performance in the FACIT population ${ }^{(12)}$, did not have any effect on leucocyte global DNA methylation status (A. Jung, Y. Smulders, P. Verhoef, F. J. Kok, H. Blom, R. Kok, E. Schouten, E. Kampman, J. Durga, 2010, unpublished results). This might be explained by the fact that methylation capacity is not exclusively dependent on folate status, as methyl groups may also be provided by dietary intake of methionine, or by betaine-mediated remethylation of homocysteine ${ }^{(9)}$.
To the best of our knowledge, this is the first study to investigate the relationship between leucocyte global DNA methylation and non-pathological cognitive functioning in healthy older adults. Future studies focusing on gene-specific DNA methylation patterns or short-term changes in DNA methylation status might contribute further to identifying the epigenetic mechanisms involved in cognitive functioning.

\section{Acknowledgements}

The FACIT study was supported by the Netherlands Organization for Health Research and Development (grant number 200110002), Sanquin Blood Bank (grant number 02-001), Wageningen University, and Top Institute Food and Nutrition. The author contributions were as follows: O. J. G. S. and J. D. designed the study. F. J. K., P. V. and J. D. were responsible for data acquisition and management of the FACIT study. O. J. G. S. analysed and interpreted the data and wrote the manuscript. All the authors reviewed and approved the final manuscript. None of the authors had a personal or financial conflict of interest.

\section{References}

1. Kado DM, Karlamangla AS, Huang MH, et al. (2005) Homocysteine versus the vitamins folate, $\mathrm{B}_{6}$, and $\mathrm{B}_{12}$ as predictors of cognitive function and decline in older highfunctioning adults: MacArthur Studies of Successful Aging. Am J Med 118, 161-167.

2. Costello JF \& Plass C (2001) Methylation matters. J Med Genet 38, 285-303.

3. Miller CA \& Sweatt JD (2007) Covalent modification of DNA regulates memory formation. Neuron 53, 857-869.

4. Levenson JM, Roth TL, Lubin FD, et al. (2006) Evidence that DNA (cytosine-5) methyltransferase regulates synaptic plasticity in the hippocampus. J Biol Chem 281, 15763-15773.

5. Costa E, Chen Y, Davis J, et al. (2002) REELIN and schizophrenia: a disease at the interface of the genome and the epigenome. Mol Interv 2, 47-57.

6. Gräff J \& Mansuy IM (2009) Epigenetic dysregulation in cognitive disorders. Eur J Neurosci 30, 1-8.

7. Tsankova N, Renthal W, Kumar A, et al. (2007) Epigenetic regulation in psychiatric disorders. Nat Rev Neurosci 8 , 355-367.

8. Ulrey CL, Liu L, Andrews LG, et al. (2005) The impact of metabolism on DNA methylation. Hum Mol Genet $\mathbf{1 4}$ R139-R147 (Spec No. 1). 
9. Niculescu MD \& Zeisel SH (2002) Diet, methyl donors and DNA methylation: interactions between dietary folate, methionine and choline. J Nutr 132, 2333S-2335S

10. Jacob RA, Gretz DM, Taylor PC, et al. (1998) Moderate folate depletion increases plasma homocysteine and decreases lymphocyte DNA methylation in postmenopausal women. I Nutr 128, 1204-1212.

11. Friso S, Choi SW, Girelli D, et al. (2002) A common mutation in the 5,10-methylenetetrahydrofolate reductase gene affects genomic DNA methylation through an interaction with folate status. Proc Natl Acad Sci U S A 99, 5606-5611.

12. Durga J, Van Boxtel MPJ, Schouten EG, et al. (2007) Effect of 3 -year folic acid supplementation on cognitive function in older adults in the FACIT trial: a randomised, double blind, controlled trial. Lancet 369, 208-216.

13. Drinkwater RD, Blake TJ, Morley AA, et al. (1989) Human lymphocytes aged in vivo have reduced levels of methylation in transcriptionally active and inactive DNA. Mutat Res 219, 29-37.

14. Brait M, Ford JG, Papaiahgari S, et al. (2009) Association between lifestyle factors and $\mathrm{CpG}$ island methylation in a cancer-free population. Cancer Epidemiol Biomarkers Prev 18, 2984-2991.

15. Kok RM, Smith DE, Barto R, et al. (2007) Global DNA methylation measured by liquid chromatography-tandem mass spectrometry: analytical technique, reference values and determinants in healthy subjects. Clin Chem Lab Med 45, 903-911.

16. Frosst P, Blom HJ, Milos R, et al. (1995) A candidate genetic risk factor for vascular disease: a common mutation in methylenetetrahydrofolate reductase. Nat Genet $\mathbf{1 0}$, $111-113$.

17. Ubbink JB, Hayward-Vermaak WJ \& Bissbort S (1991) Rapid high-performance liquid chromatographic assay for total homocysteine levels in human serum. J Chromatogr $\mathbf{5 6 5}$, 441-446.

18. De Bie SE (1987) Standaardvragen 1987: Voorstellen voor uniformering van vraagstellingen naar achtergrondkenmerken en interviews (in Dutch) (Standard Ouestions 1987: Proposal for Uniformization of Questions Regarding Background Variables and Interviews), 2nd ed. Leiden: Leiden University Press.

19. Washburn RA, Smith KW, Jette AM, et al. (1993) The physical activity scale for the elderly (PASE): development and evaluation. J Clin Epidemiol 46, 153-162.

20. Yuasa Y, Nagasaki H, Akiyama Y, et al. (2009) DNA methylation status is inversely correlated with green tea intake and physical activity in gastric cancer patients. Int J Cancer 124, $2677-2682$.

21. Fuso A, Nicolia V, Pasqualato A, et al. (2011) Changes in Presenilin 1 gene methylation pattern in diet-induced B vitamin deficiency. Neurobiol Aging 32, 187-199.

22. Scarpa S, Fuso A, D'Anselmi F, et al. (2003) Presenilin 1 gene silencing by $S$-adenosylmethionine: a treatment for Alzheimer disease? FEBS Lett 541, 145-148

23. Schilling E \& Rehli M (2007) Global, comparative analysis of tissue-specific promoter CpG methylation. Genomics $\mathbf{9 0}$, 314-323.

24. Cohen J (1988) Statistical Power Analysis for the Behavioral Sciences, 2nd ed. Hillsdale, NJ: Erlbaum. 